\title{
A SATISFAÇÃO E O DESEMPENHO DE USUÁRIOS DE PRÓTESES AUDITIVAS ATENDIDOS EM UM PROGRAMA DE ATENÇÃO À SAÚDE AUDITIVA
}

\section{Satisfaction and performance of hearing aids users assisted in a Hearing Health Care Program}

\author{
Aline da Silva Lopes ${ }^{(1)}$, Maristela Julio Costa ${ }^{(2)}$, Nilvia Herondina Soares Aurélio ${ }^{(3)}$, \\ Sinéia Neujahr dos Santos ${ }^{(4)}$, Ana Valéria Vaucher ${ }^{(5)}$
}

\begin{abstract}
RESUMO
Objetivo: avaliar o desempenho e a satisfação com o uso das próteses auditivas nos pacientes atendidos no convênio APAC-UFSM, após um ano de adaptação. Método: analisou-se 49 sujeitos, com perda auditiva do tipo neurossensorial ou mista, de grau leve a severo na melhor orelha, divididos em adultos e idosos. Realizou-se a pesquisa dos Limiares e Índices Percentuais de Reconhecimento de Sentenças no Silêncio e no Ruído, em campo livre, por meio do teste Listas de Sentenças em Português; e aplicou-se o questionário de satisfação, International Outcome Inventory for Hearing Aids. Realizaram-se os testes na mesma sessão, em dois momentos distintos, sendo o primeiro, sem o uso das próteses auditivas e o segundo, usando as mesmas.Resultados: houve diferença significante no desempenho dos indivíduos em ambos os grupos, no procedimento realizado no silêncio. Apesar de o desempenho com próteses auditivas não ter apresentado melhora significante no ruído, os pacientes apresentaram respostas satisfatórias no questionário. Na comparação entre os grupos, não se observou diferença significante quanto ao desempenho e satisfação. Conclusão: a melhora significante do desempenho no silêncio vai ao encontro do relato dos pacientes. O questionário evidenciou que a maioria referiu fazer uso efetivo das próteses auditivas e considerou ter um benefício muito satisfatório. Apesar de uma parcela significativa referir moderada ou bastante dificuldade residual, a maioria considerou que o uso das próteses auditivas vale muito ou bastante a pena.
\end{abstract}

DESCRITORES: Auxiliares de Audição; Testes de Discriminação da Fala; Questionários; Perda Auditiva

(1) Fonoaudióloga da Universidade Federal de Pelotas/UFPEL; Mestranda em Distúrbios da Comunicação Humana pela Universidade Federal de Santa Maria/UFSM.

(2) Fonoaudióloga; Professora Adjunta do Departamento de Fonoaudiologia da Universidade Federal de Santa Maria; Doutora em Ciências dos Distúrbios da Comunicação Humana pela Universidade Federal de São Paulo.

(3) Fonoaudióloga Clínica; Mestranda em Distúrbios da Comunicação Humana pela Universidade Federal de Santa Maria/UFSM.

(4) Fonoaudióloga Clínica; Mestre em Distúrbios da Comunicação Humana pela Universidade Federal de Santa Maria/ UFSM.

(5) Fonoaudióloga da Universidade Federal de Santa Maria/ UFSM; Mestre em Letras/Linguistica Aplicada /PUCRS.

Conflito de interesses: inexistente

\section{INTRODUÇÃO}

Segundo alguns autores ${ }^{1}$, a medida de resultados na Audiologia tem recebido maior atenção nos anos recentes devido à necessidade de mostrar o tratamento eficaz para os consumidores, analisar o custo-benefício e justificar a alocação de recursos.

No entanto, muitos audiologistas sentem que usar a tecnologia do microfone sonda para verificar se a prescrição alvo foi atingida pelas próteses auditivas é suficiente para verificar o desempenho das próteses auditivas. Então, essas técnicas tem funcionalmente servido como substituto dos testes de reconhecimento de fala para a avaliação do desempenho, apesar desta avaliação não fornecer 
informações sobre a habilidade de compreensão da fala do paciente em situações reais de escuta. A verificação das próteses auditivas é um importante componente na avaliação das próteses auditivas, mas não avalia se os objetivos prescritos são realmente apropriados para o paciente no que diz respeito a melhorias no reconhecimento de fala ou se o paciente irá se beneficiar do ganho prescrito as próteses auditivas ${ }^{2}$.

É de fundamental importância a medição da capacidade de reconhecimento da fala em situações mais próximas às reais, tais como testes audiológicos que empregam sentenças como estímulo, sendo realizados também na presença de ruído competitivo ${ }^{3}$.

Quantificações objetivas e subjetivas de melhorias no desempenho do reconhecimento de fala devem ser documentadas no processo de avaliação das próteses auditivas para verificar as melhorias ocorridas ${ }^{2}$.

Alguns autores ${ }^{4}$ consideram que no processo de seleção e adaptação de próteses auditivas, as informações obtidas por avaliações subjetivas podem ser mais significantes do que as obtidas por meio de avaliações objetivas.

Monitorar a satisfação e conhecer a percepção dos usuários de próteses auditivas é importante para avaliar os procedimentos clínicos e garantir os propósitos de qualidade dos serviços. Ao identificar os fatores que contribuem para a satisfação e ao tentar prover tais atributos aos processos envolvidos, tem-se o potencial de obter um resultado mais efetivo nos serviços de saúde ${ }^{5}$.

Assim, a partir dessas considerações, o objetivo desse estudo foi analisar o desempenho e a satisfação com o uso das próteses auditivas em pacientes adultos e idosos atendidos no convênio APAC-UFSM, após um ano de adaptação.

\section{MÉTODO}

Este estudo foi realizado no Laboratório de Próteses Auditivas (LPA) do Serviço de Atendimento Fonoaudiológico (SAF) da Universidade Federal de Santa Maria (UFSM) a partir do projeto "Pesquisa em base de dados em saúde auditiva", registrado no Gabinete de projetos do Centro de Ciências da Saúde sob o no 019731.

Participaram do estudo somente indivíduos que concordaram com a realização dos procedimentos necessários para a execução da pesquisa e assinaram o Termo de Consentimento Livre e Esclarecido, após terem recebido maiores esclarecimentos sobre o objetivo e metodologia do estudo.

Para fazerem parte da pesquisa, os indivíduos deveriam ter idade maior que 18 anos, diagnóstico audiológico de perda auditiva do tipo neurossensorial ou mista, de grau leve a severo na melhor orelha, ter capacidade de, pelo menos, detectar, sem o uso das próteses auditivas, a presença de som na intensidade máxima produzida pelo audiômetro ( $80 \mathrm{~dB})$, fazer parte do Programa de doação de próteses auditivas do Governo Federal, estar em processo de adaptação dos aparelhos auditivos e não ter qualquer fator que pudesse interferir no teste, como alterações neurológicas e/ou de fluência verbal.

O tempo de um ano de uso da amplificação foi estabelecido, pois é o prazo de garantia de conserto que as empresas oferecem aos beneficiados pelo programa, então se pretende verificar como eles agem após o término da garantia, e, além disso, observa-se na rotina de atendimentos realizados no Programa de Atenção à Saúde Auditiva da UFSM, que muitas vezes o serviço perde o contato com os pacientes após a adaptação das próteses auditivas, pois os mesmos não retornam para realizar as consultas de acompanhamento.

Os sujeitos que participaram do estudo foram avaliados no período que compreendeu o mês de outubro de 2008 a julho de 2009. Dentre 133 pacientes convocados, 67 compareceram e destes, 53 concluíram todas as avaliações.

No total, fizeram parte do estudo 49 sujeitos. Os indivíduos foram classificados em adultos, com idades entre 19 a 60 anos e idosos, com idades acima de 60 anos, critério estabelecido pela Lei № 8.842, de 4 de janeiro de 1994, a qual dispõe sobre a política nacional do idoso ${ }^{6}$.

O grupo de adultos é composto por 13 indivíduos, destes, sete mulheres com média de idade de 42,86 anos, com mínimo de 19 e máximo de 59 anos e seis homens com média de idade de 50,17 anos, com mínimo de 31 e máximo de 56 anos. Já o grupo formado por usuários idosos apresenta 36 indivíduos, sendo 13 mulheres com média de idade de 67,69 anos, com mínimo de 60 e máximo de 86 anos e 23 homens com média de idade de 70,96 anos, com mínimo de 61 e máximo de 84 anos.

Os indivíduos foram submetidos à pesquisa dos Limiares de Reconhecimento de Sentenças no Silêncio e no Ruído (LRSS e LRSR) e dos Índices Percentuais de Reconhecimento de Sentenças no Silêncio e no Ruído (IPRSS e IPRSR), em campo livre. Além disso, foi aplicado o questionário de satisfação, International Outcome Inventory for Hearing Aids (IOI-HA), com o título traduzido para o português como Questionário Internacional de Avaliação de Aparelhos de Amplificação Sonora Individual (QI - AASI). Os testes foram realizados na mesma sessão de avaliação, consistindo em dois instantes para cada sujeito. No primeiro momento, o paciente foi avaliado sem o uso das próteses auditivas (LRSS 
e LRSR, IPRSS e IPRSR) e no segundo momento, fazendo uso das mesmas (IPRSS e IPRSR).

Obteve-se o LRSS, IPRSS, LRSR e o IPRSR por meio do teste Listas de Sentenças para o Português (LSP) ${ }^{3}$, o qual é constituído por uma lista de 25 sentenças, outras sete listas com 10 sentenças e um ruído com espectro de fala. As sentenças e o ruído estão gravados em $C D$, em canais independentes.

Para obtenção das medidas, foi utilizado um audiômetro digital de dois canais, marca Damplex, modelo DA65; e um sistema de amplificação para audiometria em campo livre, modelo TA 1010. As sentenças foram apresentadas utilizando-se um CD Player na marca Britânia, modelo B5279, na opção lineout acoplada ao audiômetro.

Os pacientes foram posicionados de frente para a caixa de som, $0^{\circ}$ azimute, a um metro de distância. Inicialmente foi realizado um treinamento para que os sujeitos se familiarizassem com o teste e fossem obtidos os limiares aproximados. As dez primeiras sentenças da lista $1 \mathrm{~A}$ foram aplicadas aos indivíduos antes da obtenção dos LRSS.

Para a obtenção dos LRSR foi utilizada a mesma estratégia, porém na presença de ruído competitivo, com fala e ruído na mesma caixa de som.

A técnica para apresentação das sentenças foi baseada na estratégia denominada sequencial ou adaptativa ou ainda, ascendente-descendente ${ }^{7}$. Esta permite determinar o limiar de reconhecimento de fala, nível necessário para o indivíduo identificar de forma correta aproximadamente $50 \%$ dos estímulos apresentados.

O procedimento da pesquisa dos LRSS e LRSR consistiu na apresentação de um estímulo em uma determinada intensidade, obtida após o treino para a realização do teste. Se o indivíduo foi capaz de reconhecer corretamente o estímulo de fala apresentado, a intensidade do mesmo foi diminuída em intervalos preestabelecidos. Caso contrário, sua intensidade foi aumentada. Este procedimento foi repetido até o final da lista. O LRSR foi pesquisado com uma intensidade de ruído constante, a $65 \mathrm{~dB}$ NPS (A).

Foram utilizados intervalos de $4 \mathrm{~dB}$ até a primeira mudança no tipo de resposta e, posteriormente, os intervalos de apresentação dos estímulos passaram a $2 \mathrm{~dB}$ entre si até o final da lista ${ }^{7}$.

Já na pesquisa dos IPRSS e IPRSR, a intensidade das sentenças foi estabelecida por meio do LRSS e LRSR respectivamente, de cada indivíduo. No IPRSR, o ruído permaneceu constante a $65 \mathrm{~dB}$ NPS (A), determinando uma relação S/R particular a cada indivíduo.

As medidas em campo livre foram pesquisadas após a devida calibração do equipamento, por um profissional registrado no Inmetro $\mathrm{SP}$, a fim de estabelecer os níveis de pressão sonora da fala e ruído, tendo em vista as características do sinal de teste e as condições acústicas do ambiente. Para isso, foi utilizado Microfone B\&K $4144 \mathrm{n} / \mathrm{s}$, Medidor de Nível de Pressão Sonora B\&K 2260 n/s e Calibrador de Nível de Pressão Sonora B\&K n/s. O medidor foi posicionado em um ponto médio entre as duas orelhas, a uma distância de um metro do alto-falante.

O VU-meter do audiômetro foi colocado na posição 0 antes de cada avaliação e cada canal do CD foi calibrado separadamente, sendo que um tom puro de $1000 \mathrm{~Hz}$ foi utilizado para calibrar o canal das sentenças. $\mathrm{O}$ uso do tom puro é necessário, pois a fala é um som complexo, que apresenta uma variação de $30 \mathrm{~dB}$ entre o som mais intenso e o menos intenso, oscilando $12 \mathrm{~dB}$ acima e $18 \mathrm{~dB}$ abaixo da média ${ }^{8}$. Portanto, o uso de um som contínuo de referência mantém sempre as mesmas condições de apresentação. Já o canal do ruído foi calibrado usando o próprio ruído, gravado no outro canal.

Foi usado um medidor digital do nível de pressão sonora (NPS), marca Radio Schack periodicamente para monitorar os níveis de apresentação dos diferentes estímulos, determinando e garantindo sempre as mesmas condições acústicas no campo livre para todos os sujeitos avaliados.

O questionário de auto-avaliação para prótese auditiva - IOI-HA (International Outcome Inventory for Hearing Aids) ${ }^{9}$, foi realizado por meio de sete opções de respostas (uso diário, benefício, limitação de atividades básicas, satisfação, limitação da atividade auditiva, impacto nos outros e qualidade de vida) onde se pode documentar a satisfação com o uso das próteses auditivas, por meio da experiência do usuário.

A análise das respostas para o questionário foi realizada por meio da pontuação total, e da pontuação considerando cada questão. A pontuação vai de 1 (pior resultado) até 5 (melhor resultado) para cada questão e a pontuação máxima (soma de todas as questões) é de 35 pontos. Então, uma pontuação alta é sugestiva de uma avaliação positiva do desempenho da prótese auditiva segundo o usuário, e uma pontuação baixa adverte uma avaliação negativa ${ }^{10}$.

Este estudo foi aprovado pela Comissão de Ética em Pesquisa (CEP) da UFSM em 05/12/2006, protocolo no 23081.0.016862/2006-09.

Após o cálculo dos IPRSS e IPRSR e da pontuação no questionário IOI - HA, estes foram analisados e comparados estatisticamente. Após constatar que os dados tiveram distribuição normal a partir do teste de normalidade, foi aplicado o teste 
para a diferença entre duas amostras dependentes, o teste t pareado, para verificar se a melhora com próteses auditivas em cada grupo apresentou significância estatística e comparar os resultados entre o grupo de adultos e idosos. Também foi realizada uma análise descritiva dos dados, expondo os resultados para cada grupo, com e sem o uso das próteses auditivas, além de uma análise descritiva da distribuição das respostas para os itens do questionário IOI - HA em adultos e idosos. Foi considerado resultado significante $\mathrm{p} \leq 0,05$, com intervalo de confiança de $95 \%$. Os resultados significantes foram marcados com um asterisco $\left(^{*}\right)$.

\section{RESULTADOS}

Na Tabela 1 está apresentada uma análise descritiva dos dados, constando os valores médios, desvios padrões, valores mínimos e máximos do IPRSS e IPRSR sem e com o uso das próteses auditivas, e do resultado do questionário $\mathrm{IOI}-\mathrm{HA}$, no grupo de indivíduos adultos, idosos e no grupo como um todo.

A Tabela 2 mostra a análise do desempenho com o uso da amplificação sonora nos IPRSS e IPRSR, por meio do teste t pareado $(p \leq 0,05)$, no grupo de indivíduos adultos, idosos e no grupo como um todo.

Na Tabela 3 está apresentada a comparação dos valores médios obtidos com o uso das próteses auditivas, nos resultados dos testes IPRSS e IPRSR e na pontuação do questionário de satisfação IOI - HA, aplicados em adultos e idosos, por meio do teste t pareado $(\mathrm{p} \leq 0,05)$.

Os resultados significantes foram marcados com um asterisco $\left({ }^{*}\right)$.

Nas Figuras 1 a 7 estão evidenciadas as distribuições das respostas para as questões de 1 a 7 , respectivamente, do questionário $\mathrm{IOI}-\mathrm{HA}$, para os grupos de adultos e idosos.

Tabela 1 - Valores médios, desvios padrões, valores mínimos e máximos do IPRSS (\%) e IPRSR (\%) e do questionário IOI - HA - Análise descritiva

\begin{tabular}{|c|c|c|c|c|c|c|}
\hline Grupo (N) & Variáveis & Condição & Média & $\begin{array}{l}\text { Desvio } \\
\text { Padrão }\end{array}$ & Mínimo & Máximo \\
\hline \multirow{5}{*}{ Adultos (13) } & \multirow{2}{*}{ IPRSS } & Sem próteses & 50,77 & 28,42 & 0 & 100 \\
\hline & & Com próteses & 80 & 28,28 & 30 & 100 \\
\hline & \multirow{2}{*}{ IPRSR } & Sem próteses & 58,46 & 24,10 & 20 & 90 \\
\hline & & Com próteses & 68,08 & 26,73 & 40 & 100 \\
\hline & $\mathrm{IOI}-\mathrm{HA}$ & Com próteses & 28,94 & 4,10 & 20 & 35 \\
\hline \multirow{5}{*}{ Idosos (36) } & \multirow{2}{*}{ IPRSS } & Sem próteses & 51,67 & 16,12 & 20 & 80 \\
\hline & & Com próteses & 84,72 & 18,74 & 40 & 100 \\
\hline & \multirow{2}{*}{ IPRSR } & Sem próteses & 51,67 & 17,81 & 10 & 90 \\
\hline & & Com próteses & 55,83 & 29,31 & 0 & 100 \\
\hline & $\mathrm{IOI}-\mathrm{HA}$ & Com próteses & 29,46 & 3,78 & 21 & 35 \\
\hline \multirow{5}{*}{ Todos (49) } & \multirow{2}{*}{ IPRSS } & Sem próteses & 51,43 & 19,79 & 0 & 100 \\
\hline & & Com próteses & 83,47 & 21,46 & 30 & 100 \\
\hline & \multirow{2}{*}{ IPRSR } & Sem próteses & 53,47 & 19,64 & 10 & 100 \\
\hline & & Com próteses & 59,08 & 28,90 & 0 & 70 \\
\hline & $\mathrm{IOI}-\mathrm{HA}$ & Com próteses & 29,08 & 3,98 & 20 & 35 \\
\hline
\end{tabular}


Tabela 2 - Análise do desempenho com o uso das próteses auditivas no IPRSS e IPRSR, por meio do teste t pareado

\begin{tabular}{cccccc}
\hline Grupo (N) & & IPRSS (\%) & Valor de $\mathbf{p}$ & IPRSR (\%) & Valor de $\mathbf{p}$ \\
\hline \multirow{3}{*}{ Adultos (13) } & Sem próteses & 50,77 & \multirow{2}{*}{$0,0015^{*}$} & 58,46 & \multirow{2}{*}{0,0915} \\
& Com próteses & 80 & & 68,08 & \\
& Melhora & 29,23 & & 9,61 & \\
\hline \multirow{3}{*}{ Idosos (36) } & Sem próteses & 51,67 & \multirow{2}{*}{$0,0001^{*}$} & 51,67 & \multirow{2}{*}{0,1848} \\
& Com próteses & 84,72 & & 55,83 & \\
& Melhora & 30,06 & & 4,17 & \multirow{2}{*}{0,0732} \\
\multirow{2}{*}{ Todos (49) } & Sem próteses & 51,43 & \multirow{2}{*}{$0,0001^{*}$} & 53,47 & \\
& Com próteses & 83,47 & & 59,08 & \\
& Melhora & 32,04 & & 5,61 & \\
\hline
\end{tabular}

*Apresentaram diferença estatisticamente significante $(p \leq 0,05)$.

Tabela 3 - Comparação entre adultos e idosos, dos valores médios obtidos com o uso das próteses auditivas, nos testes IPRSS e IPRSR, e pontuação no questionário de satisfação IOI - HA, por meio do teste t pareado

\begin{tabular}{|c|c|c|c|}
\hline Variáveis & Grupo & $\begin{array}{c}\text { Valor médio de } \\
\text { melhora }\end{array}$ & Valor de $p$ \\
\hline \multirow{2}{*}{ IPRSS } & Adultos & $29,23 \%$ & \multirow{2}{*}{0,3083} \\
\hline & Idosos & $30,06 \%$ & \\
\hline \multirow{2}{*}{ IPRSR } & Adultos & $9,61 \%$ & \multirow{2}{*}{0,2662} \\
\hline & Idosos & $4,17 \%$ & \\
\hline \multirow{2}{*}{ Questionário IOI - HA } & Adultos & $29,46^{\star \star}$ & \multirow{2}{*}{0,6537} \\
\hline & Idosos & $28,94^{\star *}$ & \\
\hline
\end{tabular}

*Apresentaram diferença estatisticamente significante $(p \leq 0,05)$.

** Valores médios da pontuação no questionário IOI - HA.

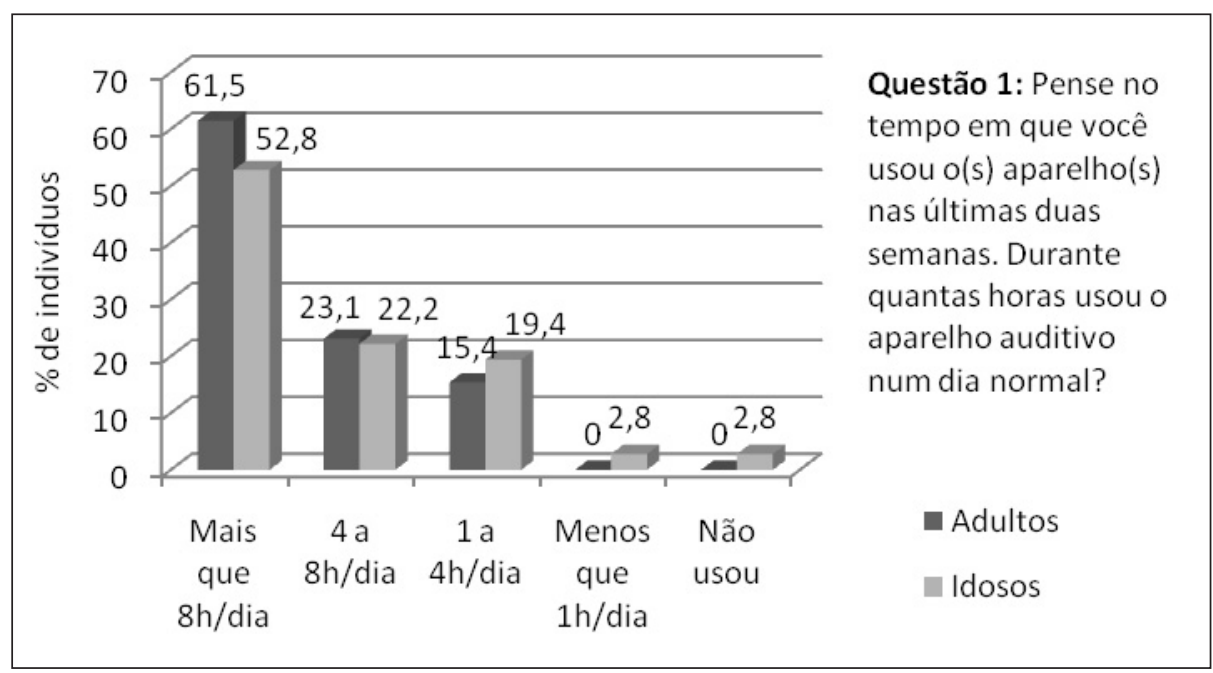

Figura 1 - Distribuição quanto ao tempo de uso diário das próteses auditivas em usuários adultos e idosos 


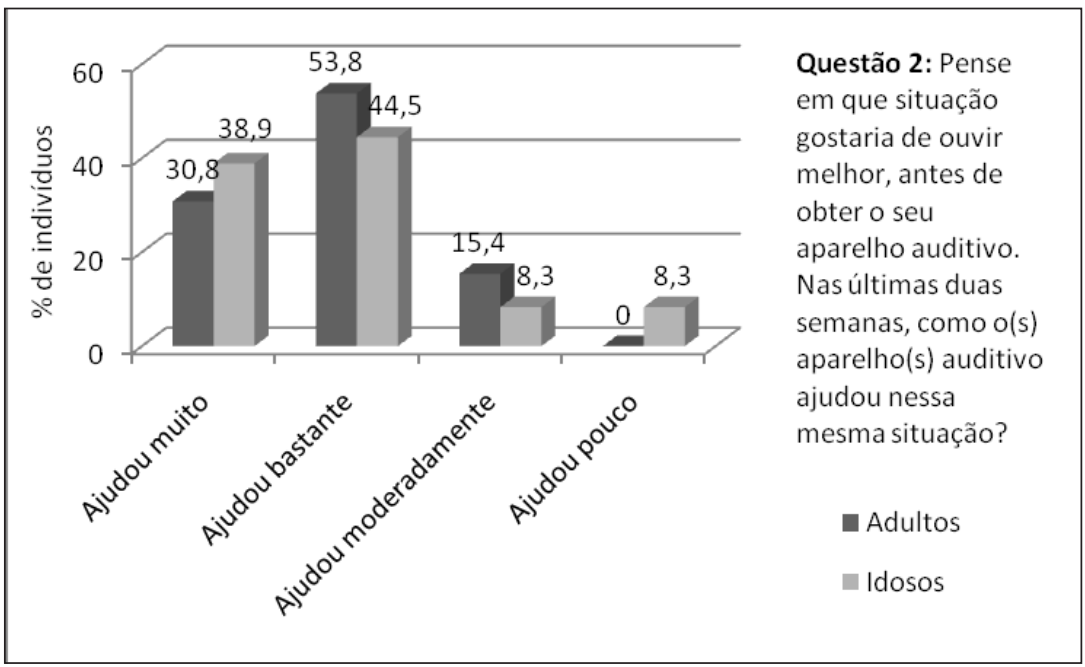

Figura 2 - Distribuição quanto ao benefício proporcionado pelo uso da amplificação sonora em usuários adultos e idosos

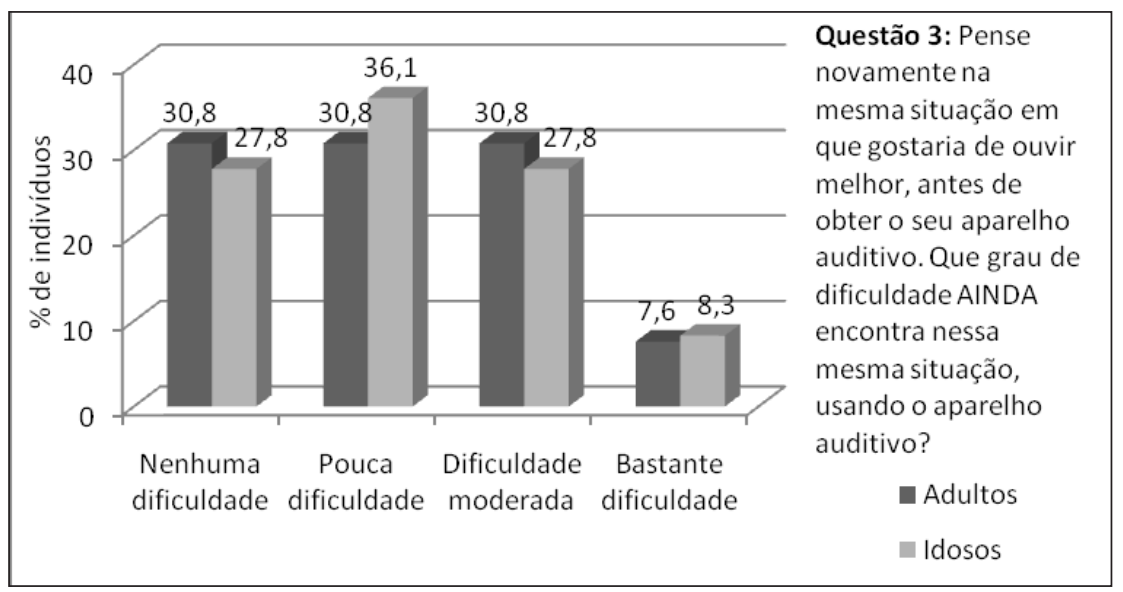

Figura 3 - Distribuição quanto à limitação da atividade auditiva em usuários adultos e idosos

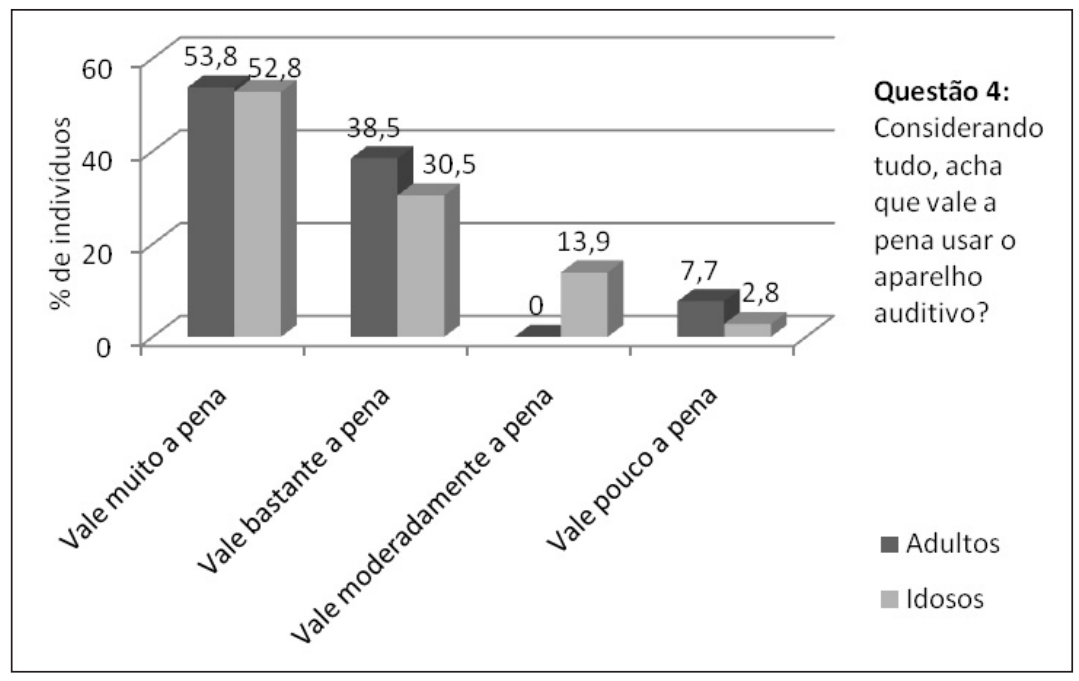

Figura 4 - Distribuição quanto ao grau de satisfação propriamente dito com o uso das próteses auditivas em usuários adultos e idosos 


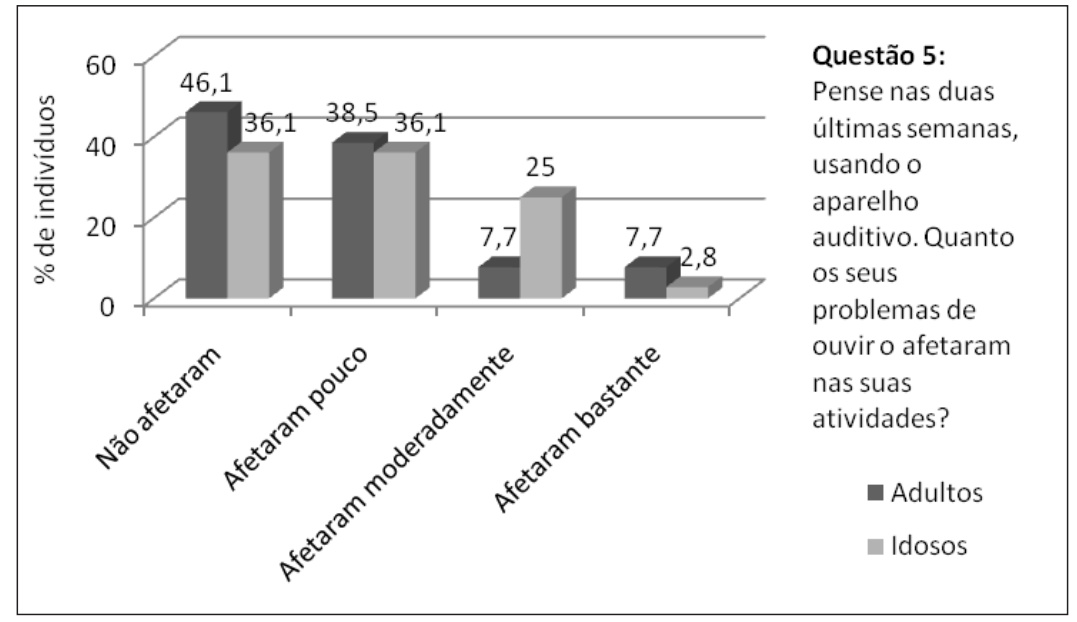

Figura 5 - Distribuição quanto à limitação residual nas atividades diárias em usuários adultos e idosos

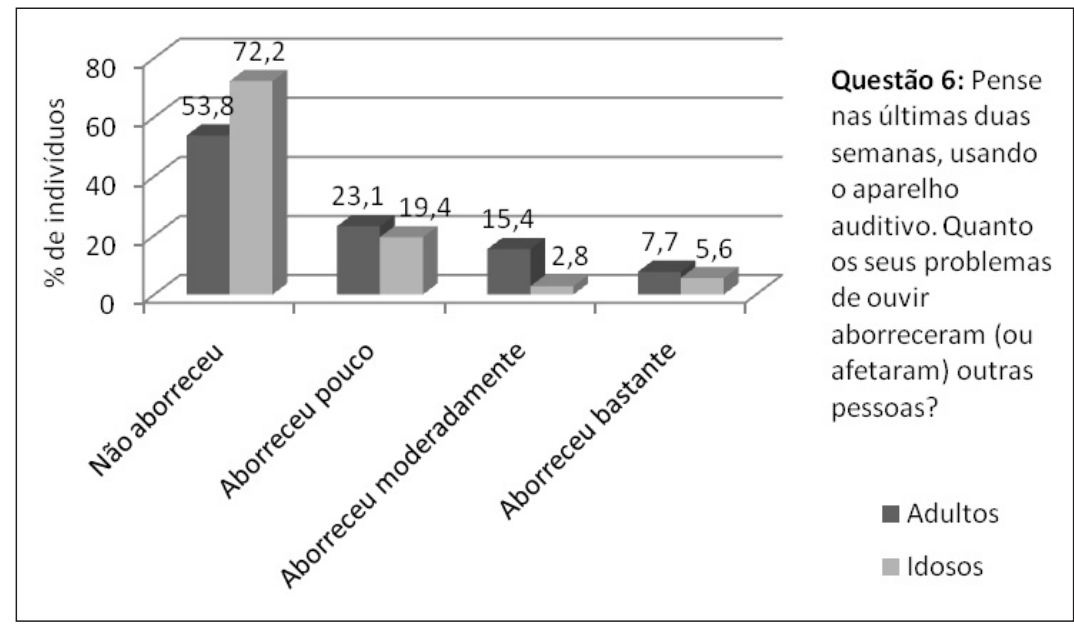

Figura 6 - Distribuição quanto ao impacto dos problemas para ouvir sobre terceiros em usuários adultos e idosos

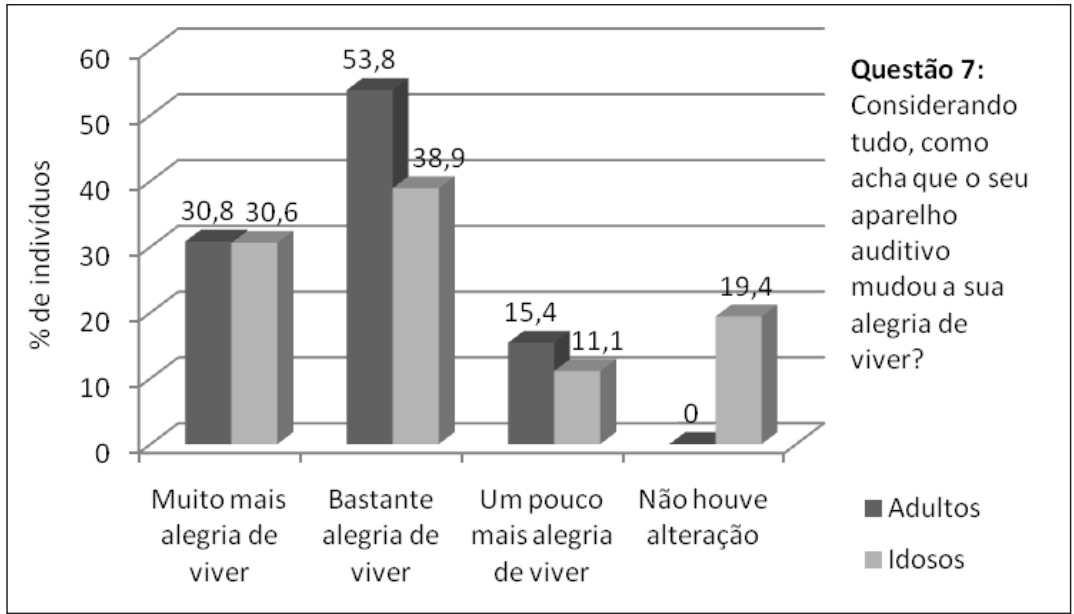

Figura 7 - Distribuição quanto à melhora na qualidade de vida proporcionada pelo uso da amplificação sonora em usuários adultos e idosos. 


\section{DISCUSSÃO}

Ao analisar estatisticamente os resultados dos IPRSS e do IPRSR no $1^{\circ}$ (sem o uso das próteses auditivas) e no $2^{\circ}$ instante (fazendo uso das próteses auditivas) foi verificada diferença significante em todos os grupos, apenas no procedimento realizado no silêncio.

Esses dados evidenciam a melhora do desempenho com o uso da amplificação sonora em situações de conversação no silêncio, indo ao encontro das queixas referidas pelos pacientes avaliados neste estudo, pois em ambiente silencioso, estes referem acentuada diferença de seu desempenho, o que não ocorre em ambientes com presença de ruído competitivo. É freqüente a queixa de a compreensão de fala na presença de ruído competitivo ser igual ou até pior com o uso das próteses.

Apesar do uso da prótese auditiva tornar os sons audíveis, a perda de audição não é recuperada. Assim, é freqüente o relato de que o paciente escuta, mas não compreende as informações e da maior dificuldade de compreender a fala em situações de escuta inadequada, como ambientes ruidosos ${ }^{11}$.

No que se refere aos valores de IPRSR, os quais são obtidos no ruído, vários estudos apontam que o mesmo é uma das maiores dificuldades encontradas pelos usuários de próteses auditivas, uma vez que a presença de ruído gera desconforto. A dificuldade é maior em situações em que há várias pessoas conversando, sendo que os participantes afirmam que sua dificuldade está em entender e não em ouvir ${ }^{12,13}$.

Ressalta-se que o progresso ocorrido até o momento tem auxiliado muito o deficiente auditivo em um aperfeiçoamento da audição para a fala, principalmente quando este se encontra em ambiente silencioso. Contudo, o desempenho que o sujeito apresenta, em ocasiões com ruído competitivo, ainda não alcançou um nível de melhora satisfatório ${ }^{14}$.

Tais dificuldades de adaptação ao uso das próteses auditivas podem se caracterizar como uma fase do processo de adaptação que envolve vários fatores: de ordem social, cultural, emocional, de questões técnicas de seleção das características da prótese ou como reflexo de uma dificuldade de percepção auditiva ${ }^{5}$.

Alguns pesquisadores ${ }^{15}$ observam que a principal queixa do deficiente auditivo se refere à dificuldade na comunicação oral, sendo que esta queixa é persistente no novo usuário de próteses auditivas que, mesmo após a amplificação, apresenta dificuldade no reconhecimento dos sons. Dessa forma, a aclimatização torna-se um grande aliado ao fonoaudiólogo e ao usuário no processo de adaptação de próteses auditivas. Não descartando a importância de todos os aspectos que envolvem o processo de seleção das próteses auditivas, como suas características eletroacústicas e a habilidade do profissional em atender as necessidades do deficiente auditivo, a aclimatização, como um fenômeno natural e inerente a esse processo, é imprescindível a uma boa adaptação, considerando uso, benefício e satisfação do usuário.

No presente estudo, todos os participantes foram adaptados há, no mínimo, um ano, período no qual a aclimatização já deveria ter ocorrido, porém, os mesmas autoras citadas acima ${ }^{15}$ ressaltam que, independentemente do tempo de uso da amplificação sonora, as dificuldades auditivas dos usuários de próteses auditivas são maiores que a de indivíduos com a audição normal.

O baixo benefício com o uso da amplificação sonora na presença de ruído competitivo pode ser justificado pela tecnologia dos aparelhos auditivos doados pelo Programa de Atenção à Saúde Auditiva, que prevê um número maior de próteses de tecnologia do tipo $A$, que possui menos recursos que possibilitem um ajuste mais personalizado; pela dificuldade na realização de acompanhamento em longo prazo, a fim de verificar se a configuração das próteses auditivas realizada inicialmente necessita de novos ajustes de acordo com a rotina diária do paciente, assim como re-orientar quanto a estratégias de facilitação de comunicação, além da verificação da necessidade de treinamento auditivo.

A criação de grupos de apoio é importante para desenvolver habilidades e confiança para interagir com outras pessoas, facilitando o processo comunicativo interpessoal. Além disso, referem que o fonoaudiólogo pode apresentar estratégias que facilitem a comunicação, reforçando as orientações sobre cuidados, manipulação e limpeza das próteses auditiva e/ou moldes auriculares, como também fornecer informações sobre audição ${ }^{16}$.

Considerando os resultados para o questionário de satisfação $\mathrm{IOI}$ - HA, em relação à questão 1, a qual analisa o tempo de uso diário das próteses auditivas, pôde-se observar que tanto no grupo de adultos quanto no de idosos, a grande maioria referiu fazer uso efetivo das mesmas (entre 4 e $8 \mathrm{~h} /$ dia e mais que $8 \mathrm{~h} /$ dia $-84,6 \%$ dos adultos e $75 \%$ dos idosos). Apesar de os indivíduos terem sido adaptados há no mínimo um ano, em ambos os grupos estudados há uma porcentagem de indivíduos que referiu ainda não fazer uso efetivo das próteses auditivas (entre 1 e 4h/dia, menos que $1 \mathrm{~h} /$ dia e não usou - $15,4 \%$ dos adultos e $25 \%$ dos idosos). Estes dados estão evidenciados na Figura 1. 
Em um estudo baseado no Profile Hearing Aid Benefit (PHAB), os autores constataram que a maior parte dos pacientes que compunham sua amostra apresentou benefício na comunicação verbal com o uso da amplificação sonora. Além disso, os mesmos observaram que o fator que mais influenciou este resultado foi o tempo de utilização da prótese auditiva, com maior benefício, quanto mais freqüente o uso da amplificação sonora ${ }^{17}$.

No que diz respeito à questão 2 , onde é analisado o benefício proporcionado pelo uso da amplificação sonora, observa-se na Figura 2 que, no grupo de usuários adultos, $84,6 \%$ referiu ter um benefício muito satisfatório, (ajudou muito e ajudou bastante) e $15,4 \%$ relatou que a prótese auditiva ajudou moderadamente. No grupo de idosos, $83,4 \%$ dos indivíduos referiram ter um benefício muito satisfatório e $16,6 \%$ relataram que o uso da amplificação ajudou moderadamente ou pouco.

Na questão 3, na qual analisa-se a limitação da atividade auditiva, em ambos os grupos estudados, verificou-se uma parcela significativa de indivíduos referindo dificuldade moderada ou bastante dificuldade (38,4\% dos adultos e $36,1 \%$ dos idosos). Estes resultados estão ilustrados na Figura 3.

Um estudo ${ }^{18}$ que pesquisou a satisfação de usuários de prótese auditiva em seu dia-a-dia revelou que os pacientes sentem-se satisfeitos, de modo geral, com o desempenho de suas próteses auditivas na vida diária, porém, os ruídos ambientais aumentados pela amplificação sonora e a performance ao telefone parecem ser os maiores problemas encontrados pelos usuários.

Considerando a questão 4, a qual verifica a satisfação propriamente dita do usuário com a amplificação sonora, em ambos os grupos, a grande maioria considera que o uso da prótese auditiva vale muito ou bastante a pena $(92,3 \%$ dos adultos e $83,3 \%$ dos idosos). A figura 4 mostra tais dados.

Outros autores ${ }^{19}$ também verificaram uma alta pontuação dos indivíduos avaliados em seu estudo no questionário $\mathrm{IOI}-\mathrm{HA}$, sugerindo atitudes favoráveis em relação ao uso de suas próteses auditivas. Os mesmos comentaram sobre a provável sensibilidade do questionário em detectar indivíduos com experiência negativa em relação às próteses auditivas.

A Figura 5 evidencia os resultados da questão 5 , onde são verificadas as possíveis limitações residuais das atividades. Pode-se observar que, no grupo de adultos, a grande maioria referiu que os problemas para ouvir não afetaram ou afetaram pouco suas atividades diárias (11 indivíduos $84,6 \%$ ), porém, no grupo composto por usuários idosos, 10 indivíduos $(27,8 \%)$ relatou que seus problemas para ouvir afetaram moderadamente ou bastante suas atividades.

Estudos corroboram estes achados, onde os participantes referem estar satisfeitos e terem benefício com o uso das próteses auditivas, embora para alguns ainda persistam dificuldades em algumas situações ${ }^{20}$.

Em relação ao impacto nas outras pessoas, caracterizado pelas respostas à questão 6 , em ambos os grupos a maioria assinalou que seus problemas para ouvir aborreceram pouco ou não causaram impacto sobre terceiros $(76,9 \%$ dos adultos e $91,6 \%$ dos idosos). Estes resultados estão evidenciados na Figura 6.

Já em outro estudo ${ }^{5}$ realizado com idosos usuários de próteses auditivas concedidas pelo Sistema Único de Saúde, na percepção dos participantes, a dificuldade para ouvir causa um "incômodo" para os familiares. As principais queixas encontradas foram em relação ao volume alto da televisão ou da necessidade de repetir o que foi falado, o que acaba irritando as pessoas com as quais convivem.

É imprescindível a implantação de um programa de reabilitação, para que o usuário e seus familiares encarem os obstáculos advindos da perda auditiva, o que pode gerar uma boa adaptação ao uso da prótese auditiva e uma facilitação na reinserção dos pacientes junto ao convívio social da família, dos amigos e dos companheiros de trabalho 11, 21, 22 .

No que diz respeito à melhora na qualidade de vida dos usuários da amplificação sonora, ilustrada na Figura 7, observa-se que tanto no grupo composto por usuários adultos quanto no de indivíduos idosos, os resultados evidenciam "alegria de viver", porém, no grupo formado por usuários idosos, os mesmos mostraram menos "alegria de viver" com o uso da amplificação sonora, quando comparados aos resultados dos adultos.

Esse achado é justificado pelo fato de os indivíduos idosos apresentarem uma percepção da qualidade de vida geral de uma maneira mais defasada em relação aos indivíduos adultos, em função de dificuldades que acompanham a presença da senilidade, tais como depressão, limitações visuais, motoras e de propriocepção, além de habilidades auditivas alteradas, as quais não estão diretamente relacionadas com o uso das próteses auditivas. Assim, essa etapa da vida envolve uma quebra das relações sociais que desencadeia isolamento do indivíduo do grupo social e perda da interação com outras pessoas, além de solidão e depressão 20. Acredita-se que nestes casos, seja realmente importante a inclusão de instrumentos, que avaliem a presença de tais fatores, complementando o questionário utilizado neste estudo. 
$\mathrm{Na}$ comparação realizada entre os grupos compostos por adultos e idosos em relação aos resultados dos procedimentos realizados com sentenças para verificar 0 desempenho das próteses auditivas e quando aplicado o questionário de satisfação IOI - HA analisado como um todo, não foi observada diferença estatisticamente significante, o que indica que, nesse estudo, adultos e idosos apresentaram o mesmo grau de satisfação.

Pode-se notar que, em ambos os grupos, apesar de o desempenho com próteses auditivas na presença de ruído competitivo não ter apresentado melhora estatisticamente significante, os pacientes apresentaram escores positivos no questionário de satisfação IOI - HA.

Em uma pesquisa realizada com idosos, os autores ${ }^{5}$ observaram que apesar das dificuldades, os participantes entrevistados percebem o benefício do uso da amplificação sonora na comunicação, diminuindo o isolamento e aumentando a autonomia nas atividades que envolvem o contato com o outro, uma vez que se sentem mais seguros em situações de comunicação. Outros estudos constataram melhora na habilidade para ouvir em diferentes situações, com repercussão positiva nas relações sociais, apesar de ainda relatarem dificuldades em algumas situações ${ }^{20}$.

Em um estudo ${ }^{23}$ que pesquisou o benefício de próteses auditivas na qualidade de vida dos usuários, constatou-se que a adaptação de próteses auditivas não é apenas fundamentada nas medidas objetivas que avaliam a audição. Atualmente, considera-se muito a avaliação do paciente em relação ao desempenho do aparelho, sua aceitação, benefício e satisfação.

A satisfação do indivíduo com relação às próteses auditivas não é sempre proporcional às mudanças observadas nas incapacidades auditivas ou mudanças de atitudes que possam refletir melhoria de qualidade de vida. Para tanto, deve-se considerar a satisfação do indivíduo que vai desde a qualidade dos serviços que o atendem até o valor final do equipamento ${ }^{24}$.

Portanto, os resultados do questionário de satisfação com escores positivos podem estar relacionados ao fato de os pacientes apresentarem sentimento de gratidão em relação ao serviço, pois receberam suas próteses auditivas gratuitamente. Alguns autores ${ }^{10}$ ressaltam que apesar dos resultados positivos obtidos na avaliação subjetiva, é preciso cautela ao afirmar tal satisfação, pois isso poderia revelar uma atitude de humildade $e$ de gratificação dos pacientes que receberam as próteses auditivas, não tendo nenhum ônus financeiro durante este processo e, possivelmente, não se achando dignos de qualquer insatisfação.
Apesar de inegável, a satisfação dos usuários depende, em grande parte, das condições físicas, emocionais, sociais e culturais, o que envolve aspectos subjetivos, particulares a cada sujeito. Os aspectos subjetivos podem explicar o fato de alguns dos participantes estarem mais satisfeitos do que outros, apesar de terem perdas auditivas e benefício com o uso de próteses auditivas similares ${ }^{20}$.

Desse modo, os questionários de auto-avaliação, assim como as medidas de ganho funcional, ganho de inserção e testes de reconhecimento de fala, devem ser utilizados conjuntamente pelos profissionais com o objetivo de avaliar o desempenho de indivíduos usuários de próteses auditivas, verificando sua adequação nas inúmeras situações de vida diária e permitindo a identificação de possíveis modificações que se façam necessárias ${ }^{20}$.

Em uma pesquisa que avaliou a qualidade de vida de adultos e idosos pós adaptação de próteses auditivas, os autores ${ }^{25}$ observaram que após o uso da prótese auditiva há melhora da qualidade de vida como um todo, evidenciando a importância do uso de prótese auditiva e do encaminhamento dos usuários para programas de adaptação e treinamento de estratégias de comunicação. Além disso, ressaltam a necessidade da criação de programas que visem a reintegração do indivíduo à sociedade, tais como o encaminhamento dos indivíduos a grupos de convivência, visando à melhora dos relacionamentos sociais.

Assim, em todos os casos o acompanhamento é imprescindível, pois a orientação e o acompanhamento são a chave do sucesso do uso efetivo do aparelho auditivo, não devendo, portanto, ser minimizada a sua importância no processo de adaptação 5, 26, 27.

\section{CONCLUSÃO}

Por meio dos resultados do presente trabalho, pode-se concluir que a melhora do desempenho com o uso da amplificação sonora foi estatisticamente significante apenas no Índice Percentual de Reconhecimento de Sentenças no Silêncio (IPRSS), tanto no grupo composto por usuários adultos quanto no de idosos, o que vai ao encontro do relato dos pacientes, os quais não referem muita dificuldade em ambientes silenciosos.

A auto-percepção da satisfação, evidenciada por meio da pontuação no questionário $\mathrm{IOI}-\mathrm{HA}$, evidenciou que em ambos os grupos, a maioria referiu fazer uso efetivo das próteses auditivas e considerou ter um benefício muito satisfatório com o uso das mesmas. Apesar de uma parcela significativa de ambos os grupos referir dificuldade 
moderada ou bastante dificuldade em relação à limitação da atividade auditiva, a grande maioria considerou que o uso das próteses auditivas vale muito ou bastante a pena.
Além disso, os questionários de auto-avaliação auxiliam no controle do desempenho dos usuários durante o processo de seleção e adaptação das próteses auditivas.

\begin{abstract}
Purpose: to assess the performance and satisfaction using hearing aids in patients assisted in the APAC-UFSM partnership, after a one-year fitting. Method: 49 subjects having mild to severe sensorineural or mixed hearing loss in the better ear were analyzed. They were divided into adults and elderly groups. The research of Sentence Recognition Threshold and Percent Indexes in Quiet and in Noise was carried out in sound field through the Portuguese Sentences Lists test; the satisfaction questionnaire, International Outcome Inventory for Hearing Aids (IOI-HA), was also applied. The tests were carried out in the same session, in two distinctive timeframes: the first one without using hearing aids, and the second one using hearing aids. Results: there was a meaningful difference in both groups regarding the procedure carried out in silence. Although the performance in noise has not resulted in meaningful improvement, the patients had positive scores in the questionnaire. When comparing both groups, no significant difference in performance and satisfaction was observed. Conclusion: the meaningful improvement in silence agrees with the patients report. The questionnaire showed that most patients referred as making effective use of the hearing aids and having a very satisfying benefit. Although some patients referred a moderate or great residual difficulty, most of them considered that using hearing aids is really worthy.
\end{abstract}

KEYWORDS: Hearing Aids; Speech Discrimination Tests; Questionnaires; Hearing Loss

\section{REFERÊNCIAS}

1. Saunders GH, Chisolm TH, Abrams HB. Measuring hearing aid outcomes - Not as easy as it seems. J Rehabil Res Dev. 2005; Supl 2; 42(4):157-68.

2. Mendel LL. Objective and Subjective Hearing Aid Assessment Outcomes. Am J Audiol. 2007; 16(2):118-29.

3. Costa MJ. Listas de sentenças em português: apresentação e estratégias de aplicação na audiologia. Santa Maria: Pallotti; 1998. p. 44.

4. Assayag FHM, Russo ICP. Avaliação subjetiva do beneficio e dos efeitos proporcionados pelo uso de amplificação sonora em indivíduos idosos. Dist Comun. 2006; 18(3):383-90.

5. Fialho IM, Bortoli D, Mendonça GG, Pagnosim DF, Scholze AS. Percepção de idosos sobre o uso de AASI concedido pelo Sistema Único de Saúde. Rev CEFAC. 2009; 11(2):338-44.

6. Brasil, Lei no 8.842 , de 4 de janeiro de 1994. Dispõe sobre a política nacional do idoso, cria o Conselho Nacional do Idoso e dá outras providências. Diário Oficial [da] República Federativa do Brasil, Brasília, DF, jan. 1994. Disponível em: < http://www.planalto. gov.br/ccivil/leis/L8842.htm> Acesso em: 26 set. 2009.
7. Levitt $H$, Rabiner LR. Use of a sequencial strategy in intelligibility testing. J Acoust Soc Am. 1967; 42(1):609-12.

8. Boothroyd A. Speech perception, sensorioneural hearing loss and hearing aid. In: Studevaker G, Hochberg I. Acustical factors affevting hearing aid performance. Boston: Allyn \& Bacon; 1993. p. 277-99.

9. Cox RM. Assessment of subjective outcome of hearing aid fitting: getting the client's point of view. Int J Audiol. 2003; 42(1):S90-6.

10. Prates LPCS, lório MCM. Aclimatização: estudo do reconhecimento de fala em usuários de próteses auditivas. Pró-Fono Revista de Atualização Científica. 2006; 18(3):259-66.

11. Miranda EC, Calais LL, Vieira EP, Carvalho LMA, Borges ACLC, lorio MCM. Dificuldades e benefícios com o uso de prótese auditiva: percepção do idoso e sua família. Rev Soc Bras Fonoaudiol. 2008; 13(2):166-72.

12. Rosa MRB, Dante G, Ribas A. Programa de orientação a usuários de prótese auditiva e questionários de auto-avaliação: importantes instrumentos para uma adaptação auditiva efetiva. Arq Int Otorrinolaringol. 2006; 10(3):220-7.

13. Lautenschlager L, Tochetto T, Gonçalves MS, Barreto DCM, Moro MP, Bolzan GP. Percepção de 
idosos socialmente ativos quanto às desvantagens causadas pela dificuldade auditiva. Einstein. 2008; 6(2):155-8.

14. Costa LP, lório MCM. Próteses auditivas: avaliações objetivas e subjetivas em usuários de amplificação linear e não-linear. Pró-Fono. 2006; 18(1):21-30.

15. Bucuvic EC, lório MCM. Benefício e dificuldades auditivas: um estudo em novos usuários de próteses auditivas após dois e seis meses de uso. Fono Atual. 2004; 29(7):19-29.

16. Vieira EP, Miranda EC, Calais LL, Carvalho LMA, lório MCM, Borges ACLC. Proposta de acompanhamento em grupo para idosos protetizados. Rev Bras Otorrinolaringol. 2007; 73(6):752-8.

17. Beamer SL, Grant KW, Walden BE. Hearing aid benefit in patients with high-frequency hearing loss. J Am Acad Audiol. 2000; 11(8):429-37.

18. Soares, D. O.; Tavares, R. A.; Ferreira, R. T.; Guglielmino, G.; Dinato, C.; Franchi, V. M. Satisfação dos usuários de prótese auditiva em seu dia-a-dia. ACTA ORL/Técnicas em Otorrinolaringologia. 2007; 25(4):290-2.

19. Cox RM, Alexander GC. The international outcome inventory for hearing aids (IOI-HA): sychometric properties of the english version. Int $\mathrm{J}$ Audiol. 2002; 41(1):30-5.

20. Costa KCF, Russo ICP, Friedman S. O sentido da deficiência auditiva e do uso de aparelhos de amplificação sonora para idosos. Rev Dist Comum. 2007; 19(3):375-87.
21. Guarinello AC, Cruz MCM. O perfil dos idosos protetizados na clínica de fonoaudiologia da Universidade Tuiti do Paraná. Fono Atual. 2006; 8(35):59-64.

22. Ruschel CV, Carvalho CR, Guarinello AC. A eficiência de um programa de reabilitação audiológica em idosos com presbiacusia e seus familiares. Rev Soc Bras Fonoaudiol. 2007b; 12(2):95-8.

23. Silman S, lório MCM, Mizhahi MM, Parra VM. Próteses auditivas: um estudo sobre seu benefício na qualidade de vida de indivíduos portadores de perda auditiva neurossensorial. Dist Comun. 2004; 16(2):153-65.

24. Magni C, Armentano JN, Moreira OS, Winter EW. Investigação do grau de satisfação entre usuários de amplificação monoaural e binaural. Dist Comun. 2005; 17(3):323-32.

25. Teixeira, A. R.; Almeida, L. G.; Jotz, G. P.; De Barba, M. C. Qualidade de vida de adultos e idosos pós adaptação de próteses auditivas. Rev Soc Bras Fonoaudiol. 2008; 13(4):357-61.

26. Freitas, C.D.; Costa, M. J. Processo de adaptação de próteses auditivas em usuários atendidos em uma instituição pública federal - Parte I: resultados e implicações com o uso da amplificação. Rev Bras Otorrinolaringol. 2007; 73(1):744-51.

27. Ruschel, C. V.; Carvalho, C. R.; Guarinello, A. C. Reabilitação auditiva em idosos. Rev Soc Bras Fonoaudiol. 2007a; 12(2):95-8.

doi: 10.1590/S1516-18462011005000005

RECEBIDO EM: 21/05/2010

ACEITO EM: 04/08/2010

Endereço para correspondência:

Aline da Silva Lopes

Rua Santa Cruz, 1340, apto 201

Pelotas - RS

CEP: 96015-710

E-mail: lopezas@gmail.com 